\title{
Riociguat for chronic thromboembolic pulmonary hypertension and pulmonary arterial hypertension: a phase II study
}

\author{
H.A. Ghofrani*,++, M.M. Hoeper ${ }^{\#,++}$, M. Halank ${ }^{\ddagger}$, F.J. Meyer ${ }^{+}$, G. Staehler ${ }^{\S}$, J. Behr ${ }^{f}$, \\ R. Ewert**, G. Weimann ${ }^{\# \#}$ and F. Grimminger*, on behalf of the study investigators ${ }^{* \|}$
}

ABSTRACT: We assessed the therapeutic potential of riociguat, a novel soluble guanylate cyclase stimulator, in adults with chronic thromboembolic pulmonary hypertension (CTEPH; $n=42)$ or pulmonary arterial hypertension $(\mathrm{PAH} ; n=33)$ in World Health Organization (WHO) functional class II/III.

In this 12-week, multicentre, open-label, uncontrolled phase II study, patients received oral riociguat $1.0-2.5 \mathrm{mg}$ t.i.d. titrated according to systemic systolic blood pressure (SBP). Primary end-points were safety and tolerability; pharmacodynamic changes were secondary end-points.

Riociguat was generally well tolerated. Asymptomatic hypotension (SBP $<90 \mathbf{m m H g}$ ) occurred in 11 patients, but blood pressure normalised without dose alteration in nine and after dose reduction in two. Median 6-min walking distance increased in patients with CTEPH (55.0 m from baseline $(390 \mathrm{~m}) ; \mathrm{p}<0.0001)$ and PAH $(57.0 \mathrm{~m}$ from baseline $(337 \mathrm{~m}) ; \mathrm{p}<0.0001)$; patients in functional class II or III and bosentan pre-treated patients showed similar improvements. Pulmonary vascular resistance was significantly reduced by $215 \mathrm{dyn} \cdot \mathrm{s} \cdot \mathrm{cm}^{-5}$ from baseline (709 dyn.s. $\left.\mathrm{cm}^{-5} ; \mathrm{p}<0.0001\right) .42(56 \%)$ patients were considered to have experienced drug-related adverse events (AEs; 96\% mild or moderate). Dyspepsia, headache and hypotension were the most frequent AEs. Study discontinuation because of AEs was $4 \%$.

These preliminary data show that riociguat has a favourable safety profile and improves exercise capacity, symptoms and pulmonary haemodynamics in CTEPH and PAH. Randomised controlled trials are underway.

KEYWORDS: Chronic thromboembolic pulmonary hypertension, clinical study, phase II, pulmonary arterial hypertension, riociguat, soluble guanylyl cyclase

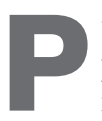

ulmonary hypertension encompasses a disparate collection of disease subtypes. Pulmonary arterial hypertension (PAH) is the best characterised of these [1], but its aetiology is still incompletely understood. Several mediators are involved in the pathogenesis of PAH; bioavailability of endogenous vasodilators such as nitric oxide and prostacyclin is reduced, and production of vasoconstrictors such as endothelin-I is increased [2]. The introduction of phosphodiesterase- 5 inhibitors, prostacyclins, and endothelin-receptor antagonists to target these signalling pathways has improved the survival of patients with PAH [3] and, although their long-term prognosis remains extremely poor, large-scale registries report that the 1 -yr survival rate is $\sim 90 \%$ [4] and about half now survive 5 yrs from initial diagnosis [5].

Similar survival trends have been seen in patients with another subtype of pulmonary hypertension, chronic thromboembolic pulmonary hypertension $(\mathrm{CTEPH})$, most likely because of the use of pulmonary endarterectomy in specialised centres. CTEPH is characterised by the presence of thrombi in the pulmonary artery. Pulmonary endarterectomy is the treatment of choice for proximal (major vessel) CTEPH, and improves symptoms and survival rates [6], but a substantial number of patients with CTEPH are not candidates for pulmonary endarterectomy (those with distal CTEPH or severe concomitant illness), or suffer persistent or recurrent pulmonary hypertension after pulmonary endarterectomy $[7,8]$. Pathophysiological similarities between CTEPH and PAH suggest that therapies targeting aberrant signalling in PAH may also be beneficial in CTEPH. However, although uncontrolled studies of several PAH therapies in patients with CTEPH have shown improvement from baseline in

\section{AFFILIATIONS}

*Dept of Internal Medicine University Hospital Giessen and Marburg, Giessen,

\#Dept of Respiratory Medicine, Hannover Medical School, Hannover, 'Medical Clinic 1/Pneumology, University Hospital Carl Gustav Carus, Dresden,

+Dept of Internal Medicine III, Medical University Clinic, Heidelberg,

${ }^{\S}$ Medical Clinic I, Loewenstein Clinic gGmbH, Loewenstein,

${ }^{f}$ Dept of Internal Medicine I,

Grosshadern Clinic, Ludwig Maximilian University of Munich, Munich,

${ }^{* *}$ Centre for Internal Medicine B, Ernst-Moritz-Arndt-University of Greifswald Clinic, Greifswald and

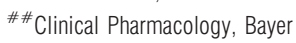
Schering Pharma AG, Wuppertal, Germany.

"A full list of the study investigators and their affiliations can be found in the Acknowledgements section.

${ }^{++}$These authors contributed equally to the study.

\section{CORRESPONDENCE}

H.A. Ghofrani, Dept of Internal Medicine, Medical Clinic II/N, University Hospital Giessen and Marburg GmbH, Klinikstrasse 36, 35392 Giessen, Germany E-mail: ardeschir.ghofrani@ innere.med.uni-giessen.de

Received:

Nov 172009

Accepted after revision: April 212010

First published online: June 072010

European Respiratory Journal Print ISSN 0903-1936 Online ISSN 1399-3003 
haemodynamics and exercise capacity, randomised placebocontrolled trials have yielded inconclusive results $[9,10]$.

Further progress in the management of CTEPH and PAH is likely to be driven by the availability of drugs with a novel mode of action. One new class of drug currently in development directly targets the nitric oxide receptor, soluble guanylate cyclase (sGC). Such sGC stimulators show a dual mode of action: they increase the sensitivity of sGC to endogenous bioavailable nitric oxide, and mimic those effects when nitric oxide is absent or scarce [11, 12]. Riociguat (BAY 63-2521) is the first member of this class to enter clinical development. Orally administered riociguat has been shown to have beneficial effects on pulmonary haemodynamics, right heart hypertrophy, and remodelling of the pulmonary vasculature in rat and mouse models of pulmonary hypertension [11]. A phase I study of 58 healthy, white male volunteers (18$45 \mathrm{yrs}$ of age) showed that single oral doses $(0.25-5 \mathrm{mg})$ of riociguat were readily absorbed and well tolerated [13]. Adverse events (AEs) were reported by $50 \%$ of the subjects, all of which were mild or moderate. No serious or lifethreatening events were reported and all but one case was resolved by study completion. A subsequent proof-of-concept study was carried out in 19 patients with CTEPH or PAH. A pilot dose titration study determined the dose of riociguat that gave the maximum clinical effect without compromising safety and tolerability. Patients then received a single, oral dose ( 1 or $2.5 \mathrm{mg}$ ) following an overnight fasting period $(\geqslant 8 \mathrm{~h})$. Pharmacodynamic parameters were evaluated at baseline, and during and after a 10-min nitric oxide inhalation period. Riociguat was administered when the haemodynamic parameters returned to baseline. Results from the proof-of-concept study showed that riociguat had a favourable safety profile, and significantly improved pulmonary haemodynamic parameters and cardiac index in patients with pulmonary hypertension, in a dose-dependent manner, and to a greater extent than nitric oxide. Mean pulmonary arterial pressure $\left(\bar{P}_{\mathrm{pa}}\right)$ was reduced by $\leqslant 40 \%$ [14]. Results of the proof-ofconcept study indicated that, as is the case for a number of currently available PAH therapies, individualised dose titration may be the most appropriate way to utilise the potential benefits of riociguat.

We present the results of a phase II study designed to assess the therapeutic potential of 12 weeks of oral riociguat treatment titrated according to systolic blood pressure (SBP) in patients with CTEPH or PAH in terms of functional as well as haemodynamic improvement, and to characterise further the safety and tolerability of this novel therapy.

\section{METHODS}

\section{Study design and patients}

This prospective, open-label, dose-titration phase II study was conducted at 15 specialist centres for the management of pulmonary hypertension in Germany; patients were enrolled and treated from February 12, 2007 to April 23, 2008. Ambulatory patients aged 18-75 yrs, who were diagnosed with CTEPH or PAH in World Health Organization (WHO) functional class II or III, were enrolled if, $<4$ weeks before the start of the study, diagnostic right heart catheterisation [15] indicated that they had $\bar{P}_{\text {pa }}>25 \mathrm{mmHg}$ and mean pulmonary vascular resistance (PVR) $>300 \mathrm{dyn} \cdot \mathrm{s} \cdot \mathrm{cm}^{-5}$.
Patients with CTEPH were eligible for inclusion if they had technically inoperable (i.e. distal) CTEPH, were deemed inoperable owing to concomitant diseases or had refused an operation. In addition, their diagnosis was established by at least two of the following: perfusion-ventilation scan, spiral computed tomography, magnetic resonance angiography or invasive pulmonary angiography (contrast medium injected into pulmonary arteries via catheter). Patients with severe systemic arterial hypertension (SBP $>200 \mathrm{mmHg}$ or diastolic blood pressure $>120 \mathrm{mmHg}$ ), systemic SBP $<100 \mathrm{mmHg}$, acute or chronic left heart failure, significant renal insufficiency (creatinine $>2 \mathrm{mg} \cdot \mathrm{dL}^{-1}(177 \mu \mathrm{M})$ or proteinuria $>1 \mathrm{~g} \cdot$ day $^{-1}$ ) or hepatic insufficiency (bilirubin $>2.5 \mathrm{mg} \cdot \mathrm{dL}^{-1}$ $(43 \mu \mathrm{M}))$ were excluded. Patients taking endothelin-receptor antagonists were allowed to continue this treatment, but those taking phosphodiesterase- 5 inhibitors or prostacyclin derivates were excluded. Diuretics, oral anticoagulants, digitalis, calcium channel blockers and oxygen supplementation were permitted.

Local institutional review boards and independent ethics committees approved the study protocol, and written informed consent was obtained from all patients.

\section{Procedures}

The primary end-points of the study were the safety, tolerability and feasibility of individual titration of riociguat according to peripheral SBP. Patients received an immediaterelease tablet of riociguat (Bayer Schering Pharma AG, Wuppertal, Germany) t.i.d. for 12 weeks, titrated in $0.5-\mathrm{mg}$ increments at 2-week intervals from a starting dose of $1 \mathrm{mg}$ to a maximum of $2.5 \mathrm{mg}$ t.i.d. Trough SBP was measured $1 \mathrm{~h}$ before administration of the morning dose of riociguat. As specified by the protocol, BP measurements were taken using a noninvasive method after a resting period of $20 \mathrm{~min}$. The methods used to take BP measurements were at the discretion of the physician and were not standardised (consistent between the study centres). The dose of riociguat was increased if trough peripheral SBP was $>100 \mathrm{mmHg}$, maintained if SBP was $90-100 \mathrm{mmHg}$ and reduced if SBP was $<90 \mathrm{mmHg}$ without symptoms of hypotension (such as dizziness or syncope); if SBP was $<90 \mathrm{mmHg}$ with symptoms of hypotension, treatment was temporarily discontinued for $24 \mathrm{~h}$ and restarted at a $0.5-\mathrm{mg}$ lower dose. At the end of the study, patients had the option to enter a long-term extension phase.

Hypotension (SBP $<90 \mathrm{mmHg}$ ) was recorded as measured by the investigator and other AEs were recorded if reported by patients spontaneously or in response to open, nondirected questioning. All treatment-emergent AEs were graded by the investigator with respect to intensity (mild, moderate or severe) and likely relationship to study medication. Any event that was fatal or life-threatening, required hospitalisation or prolongation of existing hospitalisation, resulted in persistent or significant disability or incapacity, or constituted a congenital anomaly or birth defect, was considered a serious AE. Vital signs, ECG findings and laboratory parameters were evaluated every 2 weeks. Laboratory parameters included blood gases, haematology, clinical chemistry and spontaneous urine composition. 
Secondary end-points of the study (assessed in the perprotocol population) were change from baseline (week 0) in exercise capacity (6-min walk distance (6MWD)), modified Borg dyspnoea score and WHO functional class (assessed after 12 weeks). Change in cardiopulmonary haemodynamics was also assessed after 12 weeks in patients who agreed to a second procedure; results of the diagnostic right catheterisation performed in the 4 weeks before study entry were taken as the baseline. Direct measurements included $\bar{P}$ pa, right atrial pressure, pulmonary capillary wedge pressure, mean arterial pressure and cardiac output (averaged from three measurements performed and calculated using a thermodilution device). Calculated measurements included body surface area, pulmonary vascular resistance, systemic vascular resistance and cardiac index.

\section{Statistical methods}

No formal statistical sample size estimation was performed in this exploratory study. Safety analyses were based on all patients who received at least one dose of study medication. Efficacy analyses were performed in patients who completed the study (per-protocol population). SAS ${ }_{\mathbb{R}}$ version 9.1 statistical software (SAS Institute, Cary, NC, USA) was used for data analysis. Changes from baseline in continuous pharmacodynamic variables were calculated from paired data and are presented as median (interquartile range). A Wilcoxon signedrank test was performed on these changes and a $p$-value of $<0.05$ was accepted as statistically significant.

\section{RESULTS}

\section{Patient demographics and drug dosing}

In total, 75 patients (42 with CTEPH and 33 with $\mathrm{PAH}$ ) received at least one dose of riociguat and were valid for safety analysis. Subject age was 63.0 (50-70) yrs, 41 (55\%) were female, and all were white. Six $(8 \%)$ patients were stable on treatment with the endothelin-receptor antagonist bosentan before the study; there was no ongoing or prior exposure to other classes of drug for PAH. The 12-week study was completed by 72 patients $(96 \% ; 41$ CTEPH and $31 \mathrm{PAH}$ ); their baseline demographics and clinical characteristics are shown in table 1 . As could be expected, the patients with PAH appeared to have worse baseline haemodynamics and exercise capacity than those with CTEPH. This has also been shown in registry data [16] and is unlikely to affect the interpretation of the study results. The use of concomitant medications, such as calcium channel blockers, diuretics and oxygen, at baseline was largely similar between the CTEPH and PAH subgroups (table 2). The incidence of oral anticoagulant use was slightly higher in the CTEPH group (92.7\%) than in the PAH group (71.0\%).

At the end of the study, $52(72 \%)$ patients were receiving at least the maximum dose of riociguat $(\geqslant 2.5 \mathrm{mg}$ t.i.d.) and four $(5 \%)$ were at the starting dose $(1 \mathrm{mg}$ t.i.d.); only one patient (1\%) ended the study on $0.5 \mathrm{mg}$ t.i.d. The riociguat dose was reduced by $0.5 \mathrm{mg}$ because of asymptomatic hypotension in two patients. Dose titration was associated with only a slight decrease in median SBP, from $120.0(110.0-130.0) \mathrm{mmHg}$ at baseline to $110.5(106.0-126.0) \mathrm{mmHg}$ at week $12(\mathrm{p}=0.0067)$,

TABLE 1 Baseline demographic and clinical characteristics of patients completing 12 weeks of riociguat treatment

\begin{tabular}{|c|c|c|c|}
\hline & CTEPH & PAH & Total \\
\hline Subjects & 41 & 31 & 72 \\
\hline Age yrs & $63.0(56.0-70.0)$ & $62.0(49.0-69.0)$ & $63.0(50.0-70.0)$ \\
\hline Ethnic origin white \% & $41(100)$ & $31(100)$ & $72(100)$ \\
\hline Females \% & $18(44)$ & $21(68)$ & $39(54)$ \\
\hline Body mass index $\mathrm{kg} \cdot \mathrm{m}^{-2}$ & $24.7(23.3-28.1)$ & $25.8(23.4-30.5)$ & $24.8(23.4-29.3)$ \\
\hline \multicolumn{4}{|l|}{ Functional status } \\
\hline \multicolumn{4}{|l|}{ WHO functional class } \\
\hline । & $0(0)$ & $0(0)$ & $0(0)$ \\
\hline$\|$ & $10(24)$ & $5(16)$ & $15(21)$ \\
\hline III & $31(76)$ & $25(81)$ & $56(78)$ \\
\hline Mean pulmonary arterial pressure $\mathrm{mmHg}$ & $44.0(38.0-51.0)$ & $48.0(36.0-55.0)$ & $45.5(38.0-53.0)$ \\
\hline Pulmonary vascular resistance dyn $\cdot \mathrm{s} \cdot \mathrm{cm}^{-5}$ & $686(516-859)$ & $836(511-1173)$ & 727 (513-996) \\
\hline Pulmonary capillary wedge pressure $\mathrm{mmHg}$ & $8.0(5.0-12.0)$ & $8.0(5.0-10.0)$ & $8.0(5.0-11.0)$ \\
\hline Systemic vascular resistance ${ }^{\#}$ dyn $\cdot \mathrm{s} \cdot \mathrm{cm}^{-5}$ & $1721(1346-2067)$ & $1744(1456-2275)$ & $1730(1368-2143)$ \\
\hline Cardiac index $L \cdot \min ^{-1} \cdot \mathrm{m}^{-2}$ & $2.31(1.94-2.68)$ & $2.13(1.84-2.50)$ & $2.19(1.92-2.54)$ \\
\hline Pulmonary/systemic vascular resistance ${ }^{\#} \%$ & $44.1(34.3-50.2)$ & $48.0(32.2-58.7)$ & $45.7(33.3-52.4)$ \\
\hline
\end{tabular}

Data are presented as $n, n$ (\%) or median (interquartile range). CTEPH: chronic thromboembolic pulmonary hypertension; PAH: pulmonary arterial hypertension; WHO: World Health Organization. ${ }^{\#}$ : measurements required to calculate baseline systemic vascular resistance were available for 66 patients $(C T E P H n=40 ; P A H n=26)$. 


\begin{tabular}{|c|c|c|c|c|}
\hline \multirow[t]{2}{*}{ TABLE 2} & \multicolumn{4}{|c|}{$\begin{array}{l}\text { Concomitant medication use at baseline in } \\
\text { patients completing } 12 \text { weeks of riociguat } \\
\text { treatment }\end{array}$} \\
\hline & & CTEPH & PAH & Total \\
\hline \multicolumn{2}{|l|}{ Subjects } & 41 & 31 & 72 \\
\hline \multicolumn{2}{|c|}{ Any medication } & $41(100.0)$ & $30(96.8)$ & 71 (98.6) \\
\hline \multicolumn{5}{|c|}{ PAH-specific medication } \\
\hline \multicolumn{2}{|c|}{ Any medication } & $4(9.8)$ & $2(6.5)$ & $6(8.3)$ \\
\hline \multicolumn{2}{|l|}{ Bosentan } & $4(9.8)$ & $2(6.5)$ & $6(8.3)$ \\
\hline \multicolumn{5}{|c|}{ Calcium channel blockers } \\
\hline \multicolumn{2}{|c|}{ Any medication } & $6(14.6)$ & $8(25.8)$ & $14(19.4)$ \\
\hline \multicolumn{2}{|l|}{ Amlodipine } & $4(9.8)$ & $3(9.7)$ & $7(9.7)$ \\
\hline \multicolumn{2}{|l|}{ Diltiazem } & $0(0.0)$ & $1(3.2)$ & $1(1.4)$ \\
\hline \multicolumn{2}{|l|}{ Felodipine } & $0(0.0)$ & $1(3.2)$ & $1(1.4)$ \\
\hline \multicolumn{2}{|l|}{ Lercanidipine } & $0(0.0)$ & $1(3.2)$ & $1(1.4)$ \\
\hline \multicolumn{2}{|l|}{ Nifedipine } & $1(2.4)$ & $0(0.0)$ & $1(1.4)$ \\
\hline \multicolumn{2}{|l|}{ Nitrendipine } & $1(2.4)$ & $0(0.0)$ & $1(1.4)$ \\
\hline \multicolumn{2}{|l|}{ Verapamil } & $0(0.0)$ & $2(6.5)$ & $2(2.8)$ \\
\hline \multicolumn{5}{|l|}{ Diuretics } \\
\hline \multicolumn{2}{|c|}{ Any medication } & $31(75.6)$ & 26 (83.9) & $57(79.2)$ \\
\hline \multicolumn{2}{|l|}{ Furosemide } & $7(17.1)$ & $4(12.9)$ & $11(15.3)$ \\
\hline \multicolumn{2}{|c|}{$\begin{array}{l}\text { Furosemide plus spironolac- } \\
\text { tone }\end{array}$} & $1(2.4)$ & $1(3.2)$ & $2(2.8)$ \\
\hline \multicolumn{2}{|c|}{ Hydrochlorothiazide } & $6(14.6)$ & $11(35.5)$ & 17 (23.6) \\
\hline \multicolumn{2}{|l|}{ Piretanide } & $1(2.4)$ & $0(0.0)$ & $1(1.4)$ \\
\hline \multicolumn{2}{|c|}{ Spironolactone } & $15(36.6)$ & 10 (32.3) & $25(34.7)$ \\
\hline \multicolumn{2}{|l|}{ Torasemide } & $16(39.0)$ & $16(51.6)$ & $32(44.4)$ \\
\hline \multicolumn{2}{|l|}{ Xipamide } & $3(7.3)$ & $4(12.9)$ & $7(9.7)$ \\
\hline \multicolumn{5}{|c|}{ Oral anticoagulants } \\
\hline \multicolumn{2}{|c|}{ Any medication } & 38 (92.7) & $22(71.0)$ & $60(83.3)$ \\
\hline \multicolumn{2}{|c|}{ Phenprocoumone } & 38 (92.7) & $22(71.0)$ & $60(83.3)$ \\
\hline \multicolumn{5}{|l|}{ Oxygen } \\
\hline \multicolumn{2}{|c|}{ Any medication } & $12(29.3)$ & 7 (22.6) & $19(26.4)$ \\
\hline \multicolumn{2}{|l|}{ Oxygen } & $12(29.3)$ & 7 (22.6) & $19(26.4)$ \\
\hline
\end{tabular}

Data are presented as $n$ or $n(\%)$. CTEPH: chronic thromboembolic pulmonary hypertension; $\mathrm{PAH}$ : pulmonary arterial hypertension.

and this was accompanied by a minute increase in median heart rate, from $76.0(68.0-84.0)$ beats $\cdot \mathrm{min}^{-1}$ at baseline to 76.5 $(70.5-84.4)$ beats $\cdot \mathrm{min}^{-1}$ at week $12(\mathrm{p}=0.0406)$.

\section{Safety}

Three (4\%) patients discontinued the study because of AEs (one had pulmonary oedema due to drug-related unmasking of pulmonary venous occlusive disease; one had progressive right heart failure unrelated to the study drug; one with a history of multiple allergies had drug-related exanthema). AEs were reported by $65(87 \%)$ of the 75 patients who received at least one dose of riociguat (table 3 ); these were judged to be drug-related in 42 patients (56\%). The incidence of AEs was not related to the dose of riociguat. Most drug-related AEs (96\%) were mild or moderate in severity and resulted in discontinuation in only two cases. None of the 11 reported hypotensive episodes was accompanied by symptoms leading to a permanent discontinuation of study drug; nine normalised without dose alteration and in the remaining two cases, a

TABLE 3 Adverse events (AEs) experienced by patients receiving at least one dose of riociguat $(n=75)$

Treatment-emergent events in $\geqslant 4$ patients

$\begin{array}{lc}\text { Dyspepsia } & 18(24) \\ \text { Headache } & 12(16) \\ \text { Hypotension } & 11(15) \\ \text { Peripheral oedema } & 9(12) \\ \text { Tachycardia } & 9(12) \\ \text { Fatigue } & 7(9) \\ \text { Upper abdominal pain } & 7(9) \\ \text { Dizziness } & 6(8) \\ \text { Vertigo } & 6(8) \\ \text { Vomiting } & 6(8) \\ \text { Constipation } & 5(7) \\ \text { Diarrhoea } & 4(5) \\ \text { Epistaxis } & 4(5) \\ \text { Gastrointestinal infection } & 4(5) \\ \text { Nasopharyngitis } & 4(5) \\ \text { Respiratory tract infection } & 4(5) \\ \text { Syncope } & 4(5) \\ \text { Total reporting any AE } & 65(87)\end{array}$

Data are presented as n (\%)

reduced dose was well tolerated. Syncope was reported in four patients but was not considered to be drug-related because independent causes could be identified, such as episodes of coughing or exertion after the end of the 6-min walk test. None of these cases required lowering of the dose of riociguat, and the up-titration process was continued in all four patients. No syncope occurred at the highest dose of $2.5 \mathrm{mg}$ riociguat t.i.d. 11 patients experienced serious AEs, two of whom discontinued the study (one with pulmonary oedema related to pulmonary venous occlusive disease and one with progressive right heart failure). The patient with progressive right heart failure died 50 days after stopping the study medication. None of the subjects experienced treatment-emergent elevations in serum aspartate aminotransferase or alanine aminotransferase levels to more than three times the upper limit of normal, and there were no indications of drug-induced abnormalities in other laboratory parameters. There were no specific safety or tolerability concerns in patients treated with bosentan. Median changes in correct QT interval calculated using Bazett's or Fridericia's formulae (QTc Bazett and Fridericia) from baseline to any of the visits during the initial 12-week study period were clinically insignificant: the medians of $\Delta \mathrm{QTC}$ Bazett and $\Delta$ QTC Fridericia were $2.4 \mathrm{~ms}$ and $3.0 \mathrm{~ms}$, respectively, at the end of the study (i.e. following titration of riociguat to the optimal dose in each patient).

\section{Exercise capacity}

Exercise capacity increased during riociguat treatment. Significant increases in 6MWD were observed in the whole population as well as in the CTEPH and PAH subgroups (fig. 1). By the end of the study, 6MWD had improved in $86 \%$ of patients (by $>50 \mathrm{~m}$ in $54 \%$ and by $>100 \mathrm{~m}$ in $29 \%$ ). 


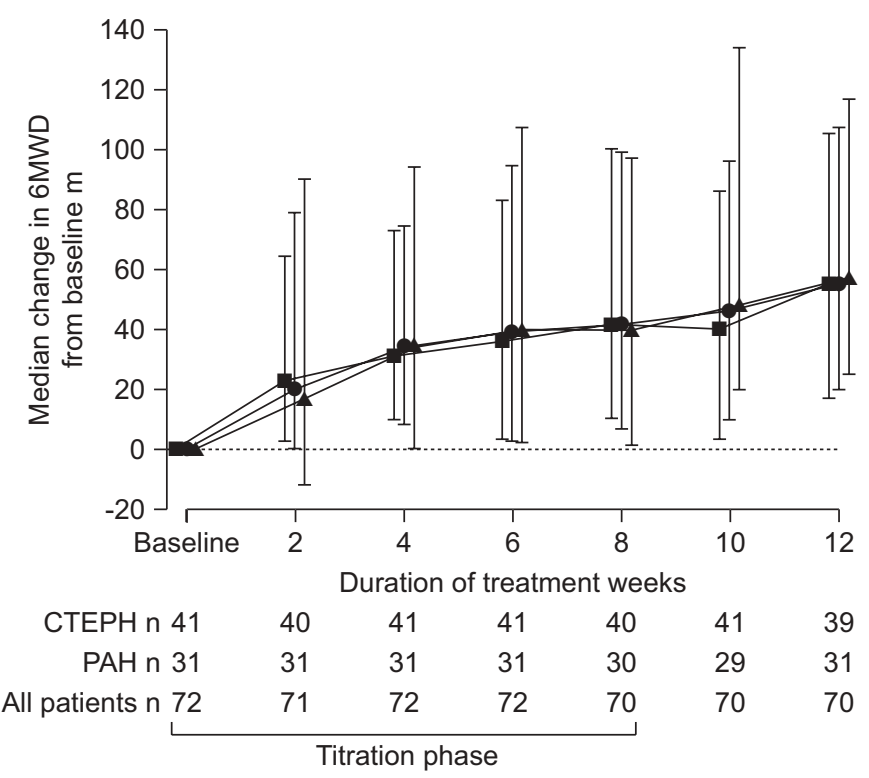

FIGURE 1. Median improvement in 6-min walk distance (6MWD) by subtype of pulmonary hypertension $(n=72)$. After 12 weeks of treatment with riociguat, significant improvements in 6MWD were observed in both the chronic thromboembolic pulmonary hypertension (CTEPH) and pulmonary arterial hypertension $(\mathrm{PAH})$ patient groups $(\mathrm{p}<0.0001)$. $\mathrm{\text {: }}$ CTEPH; $\mathbf{\Lambda}: \mathrm{PAH} ; \bullet$ : all patients. Error bars show the interquartile range.

In the total population, 6MWD was $359.0(300.0-420.0) \mathrm{m}$ at baseline. Following 12 weeks of treatment, a significant median improvement of $55(20.0-107.0) \mathrm{m}$ was observed $(\mathrm{p}<0.0001)$. Significant increases were also seen in the total population with WHO functional class II at baseline: an improvement from $423.5(396.0-511.0) \mathrm{m}$ at baseline by 71.5 $(40.0-133.0) \mathrm{m}(\mathrm{p}=0.0001)$. The improvement in patients in WHO functional class III was not as great: a significant increase of $50.0(17.0-105.0) \mathrm{m}$ from 350.0 (283.0-409.0) $\mathrm{m}$ at baseline $(p<0.0001)$. The improvement in $6 \mathrm{MWD}$ seen in patients taking riociguat with bosentan (57.5 (6.0-142.0) m; $\mathrm{p}=0.0938)$ was similar to that observed in the population taking riociguat alone $(55.0 \mathrm{~m}(22.5-106.5) \mathrm{m} ; \mathrm{p}<0.0001)$.

\section{CTEPH subgroup}

In the CTEPH group, 6MWD was 390.0 (330.0-441.0) $\mathrm{m}$ at baseline. Following 12 weeks of treatment, a significant improvement of $55(17.0-105.0) \mathrm{m}$ was observed $(\mathrm{p}<0.0001)$. An increase was also seen in patients with $\mathrm{CTEPH}$ who were WHO functional class II at baseline: an improvement from $450.0(410.0-511.0) \mathrm{m}$ at baseline by $55.0 \quad(26.0-97.0) \mathrm{m}$ $(p=0.0039)$. The improvement in patients in WHO functional class III was similar: a significant increase of 55.0 (17.0105.0) $\mathrm{m}$ from $354.5(321.0-410.0) \mathrm{m}$ at baseline $(\mathrm{p}<0.0001)$. The improvement in $6 \mathrm{MWD}$ seen in patients taking riociguat with bosentan (43.0 m (-1.5-111.0) $\mathrm{m}$; $\mathrm{p}=0.3750)$ was slightly less than in the population taking riociguat alone (55.0 (20.0105.0) $\mathrm{m} ; \mathrm{p}<0.0001)$.

\section{PAH subgroup}

Compared with patients with CTEPH, marginally greater improvements in 6MWD were observed in patients with PAH. In the PAH group, 6MWD was $337.0(215.0-406.0) \mathrm{m}$ at baseline. Following 12 weeks of treatment, a significant improvement of $57.0(25.0-117.0) \mathrm{m}$ was observed $(\mathrm{p}<0.0001)$. An increase was also seen in patients with PAH who were WHO functional class II at baseline: an improvement from 393.0 (305.0-407.0) $\mathrm{m}$ at baseline by $117.0(63.0-150.0) \mathrm{m}(\mathrm{p}=0.0625)$ The improvement in patients in WHO functional class III was not as great: a significant increase of $50.0 \mathrm{~m}(20.0-79.0 \mathrm{~m})$ from $337.0(215.0-400.0) \mathrm{m}$ at baseline $(\mathrm{p}=0.0002)$. The improvement in 6MWD seen in patients taking riociguat with bosentan $(90.0$ (35.0-145.0) $\mathrm{m} ; \mathrm{p}=0.5000)$ was greater than in the population taking riociguat alone $(57.0(25.0-115.0) \mathrm{m} ; \mathrm{p}<0.0001)$.

\section{Other exploratory outcomes}

During the 12-week study, the median modified Borg dyspnoea score improved by one point on a 10-point scale. In total, 22 out of 72 patients $(31 \%)$ showed an improvement in WHO functional class, and only one patient deteriorated (fig. 2).

In the subgroup of patients who underwent right heart catheterisation twice $(\mathrm{n}=50), \bar{P}_{\mathrm{pa}}$ had decreased significantly. We observed an improvement of $-4.5(-8.0-1.0) \mathrm{mmHg}$ from baseline $(45.0$ (37.0-53.0) $\mathrm{mmHg} ; \mathrm{p}<0.0001)$. The decrease in

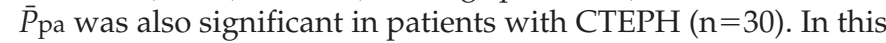
subgroup there was a median improvement of $-4.5(-7.0$ 1.0) $\mathrm{mmHg}(\mathrm{p}<0.0001)$ from baseline $(42.5(37.0-52.0) \mathrm{mmHg})$. In patients with PAH $(n=20)$, there was a median improvement of $-4.0(-15.5-1.0) \mathrm{mmHg}$ from baseline (47.5 (39.0 55.5) $\mathrm{mmHg}$ ). PVR, systemic vascular resistance and the ratio of PVR to systemic vascular resistance also decreased, while cardiac index increased (table 4). The decrease in PVR was accompanied by an increase in 6MWD in the majority of patients (fig. 3).

\section{DISCUSSION}

Both CTEPH and PAH are debilitating diseases associated with low survival rates. Although several approved pharmacological treatments now exist for $\mathrm{PAH}$, survival rates and health-related quality of life remain poor [3, 17]. Importantly, no approved pharmacological therapy is yet available for

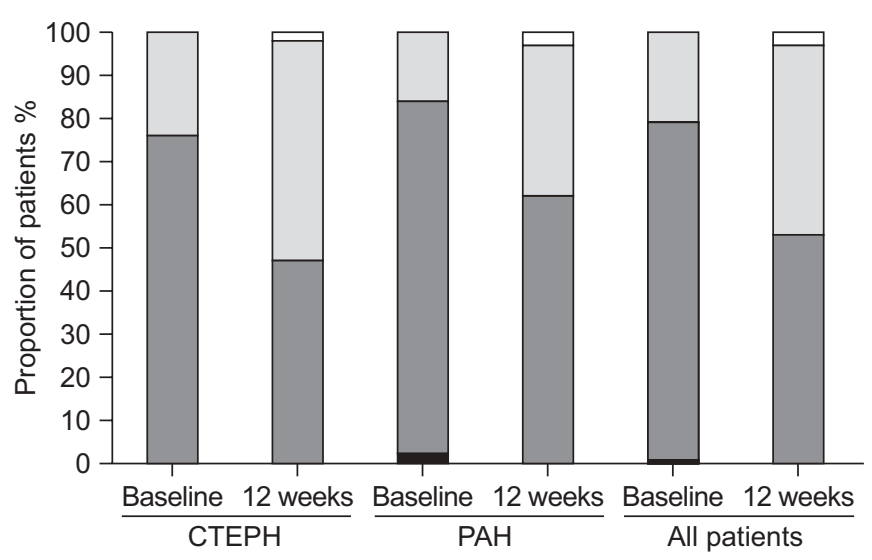

FIGURE 2. World Health Organization (WHO) functional class at baseline and at week 12 by type of pulmonary hypertension $(n=72)$. WHO functional class improved in 22 out of 72 patients and only one patient deteriorated. CTEPH: chronic thromboembolic pulmonary hypertension; PAH: pulmonary arterial hypertension. $\square$ : class I; $\mathbf{m}$ : class II; $\mathbf{\square}$ : class III; $\mathbf{\square}$ : class IV. 
TABLE 4 Change in haemodynamic parameters between baseline and week 12 for patients with week 12 measurements

\begin{tabular}{|c|c|c|c|c|c|c|}
\hline \multirow{2}{*}{$\begin{array}{l}\text { Haemodynamic } \\
\text { measurement }\end{array}$} & \multicolumn{2}{|c|}{ СТЕРН } & \multicolumn{2}{|c|}{$\mathrm{PAH}^{+}$} & \multicolumn{2}{|c|}{ All patients ${ }^{5}$} \\
\hline & Baseline & Change at week 12 & Baseline & Change at week 12 & Baseline & Change at week 12 \\
\hline $\bar{P}$ pa $\mathrm{mmHg}$ & $42.5(37.0-52.0)$ & $-4.5(-7.0-1.0)^{\#}$ & $47.5(39.0-55.5)$ & $-4.0(-15.5-1.0)^{*}$ & $45.0(37.0-53.0)$ & $-4.5(-8.0-1.0)^{\#}$ \\
\hline PVR $^{f}$ dyn $\cdot \mathbf{s} \cdot \mathbf{c m}^{-5}$ & 691 (533-854) & $-200(-288--115)^{\#}$ & $748(448-1342)$ & $-245(-560--142)^{\#}$ & 709 (521-970) & $-215(-327-117)^{\#}$ \\
\hline PCWP mmHg & $7.0(5.0-11.0)$ & $1.0(-3.0-3.0)$ & $7.5(5.5-10.5)$ & $2.0(-2.0-6.0)$ & $7.0(5.0-11.0)$ & $1.0(-3.0-4.0)$ \\
\hline PVR/SVR ${ }^{\# \# ~ \% ~}$ & $42.3(34.7-48.8)$ & $-2.9(-11.5-5.9)$ & $47.1(33.7-53.6)$ & $-5.3(-10.4-0.0)^{\star}$ & $44.8(34.2-51.9)$ & $-3.9(-11.0-2.3)^{\star *}$ \\
\hline
\end{tabular}

Data are presented as median (interquartile range). CTEPH: chronic thromboembolic pulmonary hypertension; PAH: pulmonary arterial hypertension; $\bar{P}$ pa: mean pulmonary arterial pressure; PVR: pulmonary vascular resistance; PCWP: pulmonary capillary wedge pressure; SVR: systemic vascular resistance. ${ }^{\natural}: \mathrm{n}=30 ;{ }^{+}: \mathrm{n}=20$ s: $n=50$; : data available for 48 patients (CTEPH $n=29 ;$ PAH $n=19)$; ${ }^{\# \#}$ : data available for 44 patients (CTEPH $n=28 ;$ PAH $\left.n=16\right)$. *: $p<0.05 ;{ }^{* *}: p<0.01$; ${ }^{*}: p<0.0001$.

patients with CTEPH [9]. Riociguat is the first sGC stimulator to enter clinical development. In this 12-week, multicentre, phase II trial of 75 patients with CTEPH and PAH, riociguat was well tolerated, showed a favourable safety profile, and caused strong and clinically meaningful improvements in exercise capacity and pulmonary haemodynamics in both patient subgroups.

Oral riociguat administered t.i.d. was successfully titrated from a starting dose of $1 \mathrm{mg}$ to a maximum target dose of $2.5 \mathrm{mg}$ in a majority of patients. Individualised dosing of riociguat was shown to be practicable and straightforward. SBP was measured as scheduled and it was seen that systemic vasodilation was compensated by increased cardiac output. Even though a decrease in systemic blood pressure was observed in patients as a drug-related treatment-emergent $\mathrm{AE}$, it was not clinically relevant in most cases and necessitated dose adjustment in only two patients. It is unknown if the

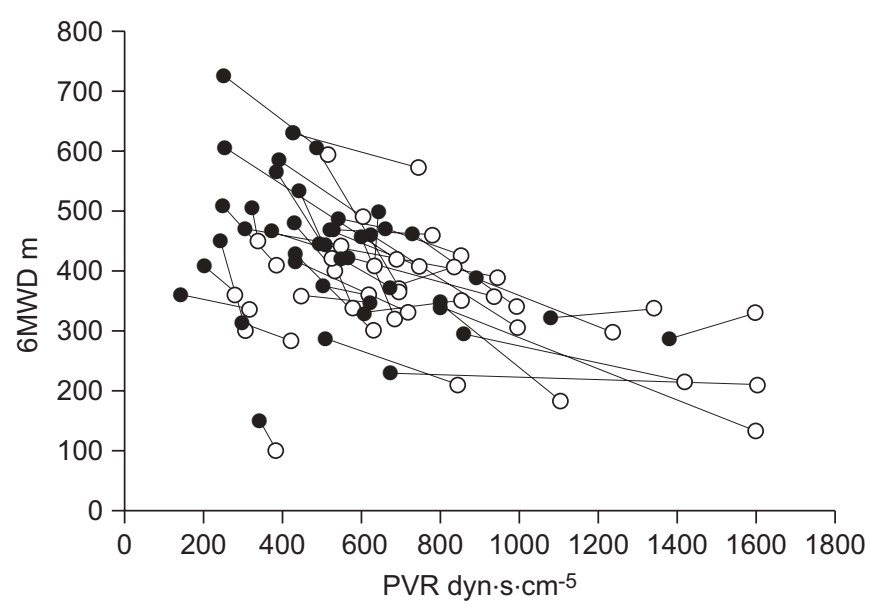

FIGURE 3. Change in 6-min walk distance (6MWD) and pulmonary vascular resistance (PVR) in individual patients after 12 weeks of treatment with riociguat $(n=48)$. Improvement in PVR was accompanied by an increase in 6MWD in the majority of patients. Paired baseline $(\bigcirc)$ and week $12(\bullet)$ values are shown for each patient. slight increase in heart rate will become clinically relevant. According to the judgment of the investigators, syncopal events were not drug-related, but careful evaluation is required to assess syncope as a potential $\mathrm{AE}$ in patients with CTEPH or PAH.

Riociguat did not cause increases in aspartate aminotransferase or alanine aminotransferase levels to more than three times the upper limit of normal in the current study. These results require confirmation in larger, longer term trials. Results from a randomised, double-blind, placebo-controlled, crossover, interaction study in 30 healthy male volunteers have previously shown that the combination of riociguat with the anticoagulant warfarin does not affect warfarin pharmacokinetics and pharmacodynamics [18]. Riociguat (2.5 mg t.i.d.) did not affect factor VII clotting activity or prothrombin time and had a favourable safety profile. The concomitant use of riociguat with existing anticoagulant therapies is therefore not expected to represent a risk.

Haemodynamics provide objective and robust direct information about the status of the pulmonary circulation. The decreases in $\bar{P}_{\text {pa }}$ (CTEPH 11\%; PAH $8 \%$ ) and PVR (CTEPH $29 \%$; PAH 33\%), and the increase in cardiac index (CTEPH $19 \%$; PAH 20\%) seen in the present study are clinically meaningful in view of those observed in previous studies of $\mathrm{PAH}$ therapies. The haemodynamic effects of riociguat warrant further investigation in randomised controlled trials.

By the end of the study, 6MWD had improved in the majority of patients and, importantly, a substantial and significant improvement in 6MWD was shown in patients with CTEPH $(55 \mathrm{~m})$ as well as in patients with $\mathrm{PAH}(57 \mathrm{~m})$. Again, these data must be confirmed in randomised, controlled studies. Interestingly, patients in WHO functional classes II and III showed similar improvements in 6MWD, suggesting that riociguat could provide marked functional improvement even in patients who have relatively mild symptoms. PAH therapies have been investigated primarily in patients who are in functional class III or IV, but observational studies indicate that there may be a clinical advantage to starting $\mathrm{PAH}$ treatment at an earlier stage of the disease $[19,20]$. 
The present study is the first to assess the therapeutic potential of sGC stimulation in pulmonary hypertension. Limitations in the design of this phase II study include its size, lack of blinding and absence of a placebo control. The relatively small proportion of patients electing to undergo a second right heart catheterisation procedure at the end of the study (67\%) should be taken into account in future studies. In addition, patients with persistent or recurrent pulmonary hypertension postpulmonary endarterectomy were not included in the current study. These patients should be considered for inclusion alongside patients with inoperable CTEPH in future studies of riociguat. Blood pressure was recorded using a noninvasive device after a resting period of $20 \mathrm{~min}$. The exact methodology was decided by the study investigator. A standardised protocol will be used for phase III studies to ensure consistency between study centres. A larger trial size and grouping of specific PAH aetiologies in future studies may permit useful comparisons of efficacy between PAH subtypes. Finally, this study, which received approval from independent ethics committees in Germany, involved newly diagnosed patients foregoing treatment with approved $\mathrm{PAH}$ therapies in order to be treated with an investigational drug. The ethics of such studies must always be carefully considered when designing the protocol, bearing in mind the potential risks in relation to the research value of the study. The delaying of treatment with approved PAH therapies is the subject of ongoing debate, particularly in the context of placebo-controlled studies [21].

Despite these limitations, the magnitude of the median improvements from baseline in 6MWD and haemodynamic parameters makes the findings promising. The favourable tolerability of riociguat and its positive effects on haemodynamics, 6MWD, WHO functional class and dyspnoea scores could potentially translate into improvements in health-related quality of life for patients, and further randomised, controlled study of riociguat in CTEPH and PAH is warranted. Data from the small number of patients taking bosentan provide the first hint that combination therapy with endothelin-receptor antagonists and riociguat may be feasible. The use of riociguat in combination with existing PAH therapies should also be investigated in future clinical studies.

The results of this phase II study suggest that $1.0-2.5 \mathrm{mg}$ t.i.d. riociguat may have a favourable safety profile and may improve the exercise capacity, symptoms and haemodynamics of patients with CTEPH and PAH. Two global, phase III clinical studies in CTEPH and PAH have recently been initiated, and clinical studies to evaluate the effect of riociguat in several other pulmonary and cardiovascular indications are underway.

\section{SUPPORT STATEMENT}

This study was supported by Bayer Schering Pharma AG, Berlin, Germany.

\section{CLINICAL TRIAL}

This study was registered with ClinicalTrials.gov indentifier NCT00454558.

\section{STATEMENT OF INTEREST}

Statements of interest for all authors can be found at www.erj. ersjournals.com $/ \mathrm{misc} /$ statements.dtl

\section{ACKNOWLEDGEMENTS}

We would like to thank the study investigators: B. Karadas (University of Giessen, Giessen, Germany); H. Wirtz and H.-J. Seyfarth (University of Leipzig, Leipzig, Germany); G. Hoeffken (University of Dresden Hospital, Dresden, Germany); H. Golpon (University of Hannover, Hannover, Germany); V. Mitrovic and B. Swidnicki (Kerckhoff-Clinic Bad Nauheim, Bad Nauheim, Germany); M. Buerke and M. Russ (University of Halle, Halle, Germany); C.F. Opitz (DRK Hospital of Berlin, Berlin, Germany); H. Leuchte (University of Munich, Munich, Germany); H. Klose and M. Karjalainen (University of Hamburg and CTC Hamburg, respectively; Hamburg, Germany); H. Wilkens (University of Saarland, Homburg, Germany); S. Rosenkranz (University of Cologne, Cologne, Germany); M. Pfeifer and T. Lange (University of Regensburg, Regensburg, Germany); S. Glaeser (University of Greifswald, Greifswald, Germany). We would also like to thank S. Unger (Bayer Schering Pharma AG, Wuppertal, Germany) for statistical analysis of the data. C. Mulligan (Oxford PharmaGenesis Ltd, Oxford, UK) provided editorial assistance, supported by Bayer Schering Pharma AG.

\section{REFERENCES}

1 Simonneau G, Galie N, Rubin LJ, et al. Clinical classification of pulmonary hypertension. J Am Coll Cardiol 2004; 43: 5S-12S.

2 McLaughlin VV, McGoon MD. Pulmonary arterial hypertension. Circulation 2006; 114: 1417-1431.

3 Galie N, Manes A, Negro L, et al. A meta-analysis of randomized controlled trials in pulmonary arterial hypertension. Eur Heart $J$ 2009; 30: 394-403.

4 Humbert M, Sitbon O, Chaouat A, et al. Pulmonary arterial hypertension in France: results from a national registry. Am J Respir Crit Care Med 2006; 173: 1023-1030.

5 Thenappan T, Shah SJ, Rich S, et al. A USA-based registry for pulmonary arterial hypertension: 1982-2006. Eur Respir J 2007; 30: 1103-1110.

6 Condliffe R, Kiely DG, Gibbs JS, et al. Improved outcomes in medically and surgically treated chronic thromboembolic pulmonary hypertension. Am J Respir Crit Care Med 2008; 177: 1122-1127.

7 Bresser P, Pepke-Zaba J, Jais X, et al. Medical therapies for chronic thromboembolic pulmonary hypertension: an evolving treatment paradigm. Proc Am Thorac Soc 2006; 3: 594-600.

8 Hoeper MM, Mayer E, Simonneau G, et al. Chronic thromboembolic pulmonary hypertension. Circulation 2006; 113: 2011-20.

9 Lang IM. Managing chronic thromboembolic pulmonary hypertension: pharmacological treatment options. Eur Respir Rev 2009; 18: $24-28$.

10 Jais X, D'Armini AM, Jansa P, et al. Bosentan for treatment of inoperable chronic thromboembolic pulmonary hypertension: BENEFiT (Bosentan Effects in iNopErable Forms of chronIc Thromboembolic pulmonary hypertension), a randomized, placebo-controlled trial. J Am Coll Cardiol 2008; 52: 2127-2134.

11 Schermuly R, Stasch JP, Pullamsetti SS, et al. Expression and function of soluble guanylate cyclase in pulmonary arterial hypertension. Eur Respir J 2008; 32: 881-891.

12 Mittendorf J, Weigand S, Alonso-Alija C, et al. Discovery of riociguat (BAY 63-2521): a potent, oral stimulator of soluble guanylate cyclase for the treatment of pulmonary hypertension. Chem Med Chem 2009; 4: 853-865.

13 Frey R, Mück W, Unger S, et al. Single-dose pharmacokinetics, tolerability and safety of the soluble guanylate cyclase stimulator BAY 63-2521; an ascending-dose study in healthy male volunteers. J Clin Pharmacol 2008; 48: 926-934.

14 Grimminger F, Weimann G, Frey R, et al. First acute haemodynamic study of soluble guanylate cyclase stimulator riociguat in pulmonary hypertension. Eur Respir J 2009; 33: 785-792. 
15 Ghofrani HA, Wiedemann R, Rose F, et al. Sildenafil for treatment of lung fibrosis and pulmonary hypertension: a randomised controlled trial. Lancet 2002; 360: 895-900.

16 Tueller C, Stricker H, Soccal P, et al. Epidemiology of pulmonary hypertension: new data from the Swiss registry. Swiss Med Wkly 2008; 138: 379-384.

17 Naeije R, Huez S. Expert opinion on available options treating pulmonary arterial hypertension. Expert Opin Pharmacother 2007; 8 2247-2265.

18 Frey R, Mück W, Kirschbaum N, et al. Warfarin pharmacodynamics and pharmacokinetics are not affected by the soluble guanylate cyclase stimulator riociguat (BAY 63-2521). BMC Pharmacol 2009; 9: Suppl. 1, P15.

19 McLaughlin VV, Shillington A, Rich S. Survival in primary pulmonary hypertension: the impact of epoprostenol therapy. Circulation 2002; 106: 1477-1182.

20 Sitbon $\mathrm{O}$, Humbert $\mathrm{M}$, Nunes $\mathrm{H}$, et al. Long-term intravenous epoprostenol infusion in primary pulmonary hypertension: prognostic factors and survival. J Am Coll Cardiol 2002; 40: 780-788.

21 Halpern SD, Doyle R, Kawut SM. The ethics of randomized clinical trials in pulmonary arterial hypertension. Proc Am Thorac Soc 2008; 5: 631-635. 